\title{
¿Se debería prohibir la integración vertical entre Isapres y Clínicas?
}

Ramiro de Elejalde. Académico FEN UAH

En Chile, a partir de 2005, la integración vertical entre seguros de salud privados
(isapres) y prestadores de salud (clinicas (isapres) y prestadores de salud (clinicas,
centros médicos, etc.) quedó prohibida por centros médicos, etc.) quedó prohibida por
ley. Sin embargo, las isapres y los prestadores han utilizado la figura del holding para santener una integración vertical de facto s salud es una empresa que es propietaria de una lsapre yun prestador pero al no ha-

ber ninguna relación directa entre ambos no va en contra de la ley.

Los holdings en el mercado de la salud en Chile son:

- Empresas Banmédica S.A.: es propietaria de las Isapres Banmédica y Vida Tres, las clinicas Santa Maria, Dávila, Vespucio Ciudad del Mar y Bío Bio, el prestador ambulatorio Vidaintegra, y el servicios

- Empresas Red Salud S.A.: es propietaría de la Isapre Consalud, las clinicas Bicentene participaín en un consorcio $y$ clinicas regionales, y el prestador ambulatorio Megasalud.

- Bupa Chile S.A.: es propietaria de Isapre Cruz Blanca, las clinicas Antofagasta, Reñaca y San José, y los prestadores ambulatorios IntegraMédica, Sonorad y Centro Dra Pilar Gazmur.

- Empresas MasVida S.A.: es propietaria de la Isapre MasVida, las clinicas El Loa Las Lilas, Isamédica, Chillán, Universitaria de Concepción y Universitaria de Puerto

De hecho, ta inice isapre abierta que ac- tualmente no es propietaria de un prestador es Isapre Colmena'.

Los holdings pueden ser legales pero van en contra del espiritu de la ley, y creemos que esta situación se deberia transparentar.
Pero la pregunta relevante es si hace sentido prohibir la integración vertical entre seguros médicos y prestadores. Entonces, ¿qué nos dice la teoría y la evidencia empirica sobre la integración vertical en mercados de salud?

La teoría encuentra efectos positivos y efectos

El efecto positivo más importante es las ganancias de eficiencia. Una ganancia de eficiencia de la integración vertical puede venir a traves de una menor sobreutilización de los servicios médicos. Por ejemplo, un prestador de salud puede tener incentivos al solicitar estudios adicionales dado que ni el ni el paciente internalizan los cos- deso integrado seguro-prestador Un mointernalicen estos costos reduciendo el número de estudios innecesarios

De hecho, en Estados Unidos existe evidencia que las empresas integradas de salud como Kaiser Permanente obtienen ganancias de eficiencia en costos.

Otras ganancias de eficiencia pueden venir a traves de mejoras en la coordinación y monitoreo entre el seguro médico y ejemplo, la implementación de sistemas de formación

Los efectos negativos se deben a efectos estratégicos de la integración que afectan emportamiento competitivo de las enpresas en el mercado.

Ll efecto estratégico más importante el el incentivo de una empresa integrada (antere gado tiene incentivos a cobrar un precio mayor a otros seguros de salud que no perecen a su holding. Al cobrar un precio mayor a otros seguros, el seguro integrado obtiene una ventaja competitiva en el merado de seguros. Al final del día, el mercado e seguros se puede volver menos competivo y con mayores primas.

tratégico de la integración vertical son las redes exclusivas: seguros de salud con una red de prestadores propios arder a consumidores.

Por último, la integración vertical puede er los acuerdos colusivos al facilrivales a través de los prestanores propios. Desafortunadamente no existe evidencia empirica que nos diga cuán importantes son estos efectos estratégicos en la práctio bajo qué condiciones pueden ser $m$ as mportantes.

LA EVIDENCIA PARA CHILE

No existen estudios para Chile que nos

ayuden a dilucidar que tan importantes

son las ganancias de eficiencia y los efeclos estrategicos de la integracion vertical. informada en base a los datos disponibles. En primer lugar, en los casos donde se han encontrado mayores ganancias de eficiencia de la integración vertical como Kaser en EEUU, los médicos reciben un salario fijo y los asegurados solamente pueden acceder a una red restringida de prestadores que son propiedad de la empresa integrada. Este no es el caso en Chile. Los seguros integrados ofrecen planes preferentes con sus propias clinicas pero tambien ofrecen planes prefentes con otras clinicas y plaelige el prestador. Esto nos hace dudar de que existan ganancias de eficiencia importantes de la integración vertical en el pais En segundo lugar, para que los efectos estratégicos sean importantes los mercados de seguro y clinicas deben ser concentrados. En la figura 1 y 2 hemos calculado el indice de concentración de HerfindahHirschman (IHH) para prestadores privados e isapres. Según los criterios de la Fiscalía Nacional Económica, ambos mercados son moderadamente concentrados (valores del THH entre 1.500 y 2.500). Por lo tanto, los efectos estratégicos pueden estar presenPor otro lado, c

eisten redes e, como mencionamos, no pres integradas tienen contratos con otras clinicas y las clinicas integradas tiene contratos con otras isapres. Con lo que el efecto estratégico de redes exclusiva no parece ser relevante.

Salud como en el caso de las fusiones hofizontales que deben ser aprobadas

IEn el pasado, Isapre Colmena era propietaria de En el pasado, lsapre Colmena era propietaria
prestadores de salud como la Clíica San Carlos de

Figura 1: Índice de Herfindahl-Hirschman (IHH) para prestadores . 1.600

1.400

1.200

1.000

포 800

600

400

20012002200320042005200620072008200920102011201220132014

- IHH por holding _ - IHH por prestador hospitalaril

Oe

Figura 2: Índice de Herfindahl-Hirschman (IHH) para el mercado de Isapres. 2001-2015. Chile.

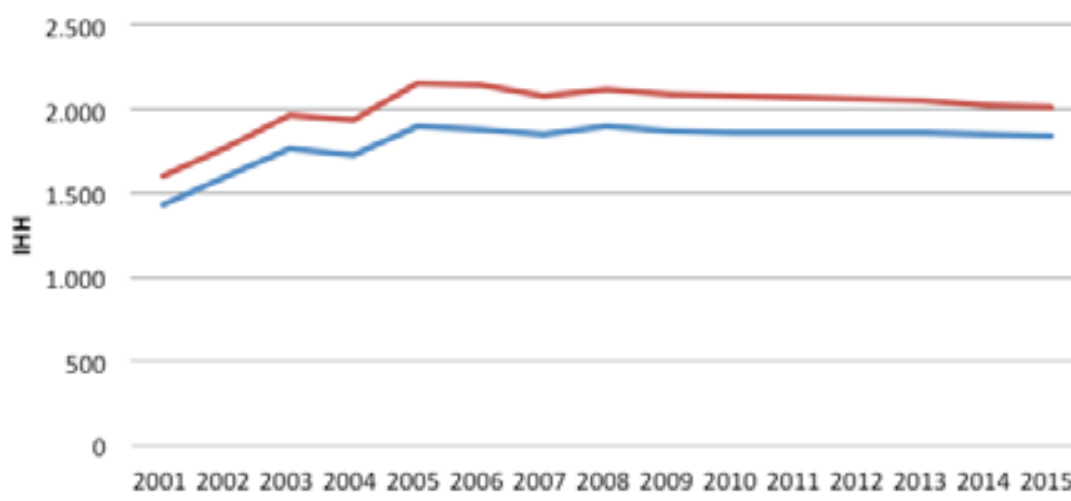
-

\section{Fuente: Superintendencia de Salud, Ministerio de Salud, Chile.}

RECOMENDACIONES DE POLITICA

No existen razones económicas para

Una politica más razonable seria requeri

Facultad: 28897366 e-mail: jrodrigu@uahurtado 